\title{
Effect of visual surrounding motion on body sway in a three-dimensional environment
}

\author{
MICHEL GUERRAZ, CLAIRE C. GIANNA, PETER M. BURCHILL, \\ MICHAEL A. GRESTY, and ADOLFO M. BRONSTEIN \\ Medical Research Council, National Hospital for Neurology and Neurosurgery, London, England
}

\begin{abstract}
Unidirectional motion of a uniplanar background induces a codirectional postural sway. It has been shown recently that fixation of a stationary foreground object induces a sway response in the opposite direction (Bronstein \& Buckwell, 1997) when the background moves transiently. The present study investigatedfactors determining this contradirectional postural response. In the experiments presented, center of foot pressure and head displacements were recorded from normal subjects. The subjects faced a visual background of $2 \times 3 \mathrm{~m}$, at a distance of $1.5 \mathrm{~m}$, which could be moved parallel to the interaural axis. Results showed that when the visual scene consisted solely of a moving background, the conventional codirectional postural response was elicited. When subjects were asked to fixate an earth-fixed foreground (window frame) placed between them and the moving background, a consistent postural response in the opposite direction to background motion was observed. In addition, we showed that this contradirectional postural response was not transient but was sustained for the $11 \mathrm{sec}$ of background motion. We investigated whether this contradirectional postural response was the consequence of the induced movement of the foreground by background motion. Although induced movement was verbally reported by subjects when viewing an earth-fixed target projected onto the moving background, the contradirectional sway did not occur. These results indicate that foreground-background separation in depth was necessary for the contradirectional postural response to occur rather than induced movement. Another experiment showed that, when the fixated foreground was attached to the head of the observer, the contradirectional sway was not observed and was therefore unrelated to vergence. Finally, results showed that the contradirectional postural response was, in the main, monocularly mediated. We conclude that the direction of the postural sway produced by a moving background in a three-dimensional environment is determined primarily by motion parallax.
\end{abstract}

Movement of visual surroundings (real or projected) induce postural adjustments in human subjects. Real visual environments used to study this phenomenon have been tilting rooms (Bles, Kapteyn, Brandt, \& Arnold, 1980), rotating disks (Dichgans, Held, Young, \& Brandt, 1972), and translating rooms (Bronstein, 1986; Lee \& Lishman, 1975). Projected visual displays have simulated moving walls, tunnels, floors, and ceilings (Dijkstra, Schöner, \& Gielen, 1994; Fluckiger \& Baumberger, 1988; Lestienne, Schoechting, \& Berthoz, 1977; van Asten, Gielen, \& Diener van der Gon, 1988). Those experiments have shown that motion of a single surface background (in the simplest case, two dimensional) induces a codirectional postural sway.

Postural adjustments in the opposite direction to motion of the surrounding (contradirectional sway) have also been reported. Bronstein and Buckwell (1997) found systematic postural adjustments in the opposite direction of display motion when a stationary object of fixation was placed in the foreground. In their experiment, a visual background was moved behind a stationary window (fore-

Correspondence should be addressed to M. Guerraz, MRC Human Movement and Balance Unit, Institute of Neurology, The National Hospital for Neurology and Neurosurgery, Queen Square, London WC1N 3BG, England (e-mail: dizzymrc@ion.ucl.ac.uk). ground) with a velocity and amplitude that created an optic flow comparable to what a standing subject would experience during spontaneous quiet sway in a threedimensional (3-D) environment. As shown in Figure 1, when an observer moves laterally while fixating a distant object, the images of nearer objects move in the direction opposite to that of head movement. When the observer fixates a nearer object, the direction of motion of the images of distant objects moves in the same direction as the head. Then, the postural adjustment in the opposite direction of background motion is consistent with the direction of image movement on the retina that a moving subject would experience in a 3-D environment. Useful in the determination of depth (see Howard \& Rogers, 1995, for review) and locomotion (Bardy, Warren, \& Kay, 1996; Warren, Kay, \& Yilmaz, 1996), motion parallax (i.e., the relative movement of images across the retina) also appears to be a potential source of information to stabilize standing posture. Since a short-duration (2-sec) stimulus was used by Bronstein and Buckwell, the aim of our first experiment was to determine whether this postural response is only a transient phenomenon or whether it can sustain for a longer period. This would parallel findings on the codirectional anteroposterior sway observed with a uniplanar moving visual environment (Lestienne et al., 1977). 


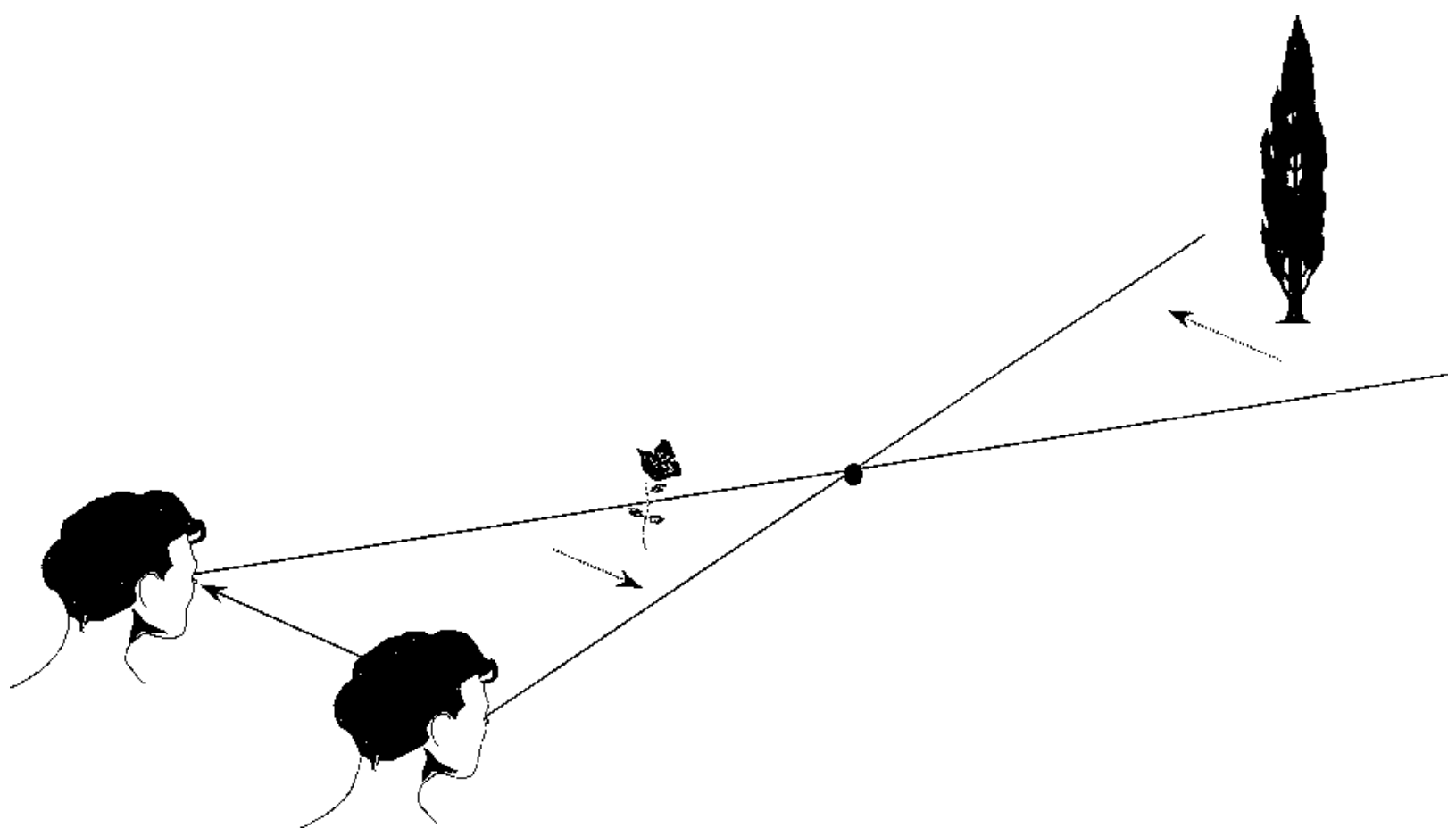

Figure 1. When the observer moves his/her head from right to left while fixating a stationary point $(\bullet)$, the image of the flower in the foreground moves from the left to the right relative to the line of sight. The image of the tree in the background moves from the right to the left, relative to the line of sight. The nearer object (flower) then appears to move in the opposite direction of head movement, whereas the distant object (tree) appears to move in the same direction as the head.

A perceived movement of the fixated foreground induced by the moving background ("induced movement"; see Reinhardt-Rutland, 1988, for review) could also be responsible for the contradirectional sway observed by Bronstein and Buckwell (1997). Van Asten et al. (1988) investigating visually induced body sway in the anteroposterior direction reported that when the central part of their linearly moving display was masked, anteroposterior sway was in the opposite direction of background motion (moving forward-backward) in $25 \%$ of the trials. Since the stationary inner part of their display was perceived as moving in the opposite direction of motion (induced movement in depth), the authors interpreted the sway opposite of motion as determined by induced movement of the stationary inner part of the display. Thus, the perceived movement of a target rather than its retinal image movement can be a relevant cue for controlling posture. Analogously, it has been shown that perceived motion can generate nystagmic eye movements with slow phases in the direction of induced movement both in monkeys (Waespe \& Schwarz, 1987) and in humans (Collewijn, Curio, \& Grüsser, 1982; Heywood, 1973; Yasui \& Young, 1975).

The present study was divided into four experiments. Experiment 1 investigated whether the contradirectional postural adjustments observed with a foreground target can be sustained for longer than 1-2 sec. The purposes of Experiments 2 and 3 were to investigate whether the contradirectional sway observed in a 3 -D environment is due to induced movement of the fixated foreground, motion parallax, or simply convergence of the eyes on a close target in front of a moving background. Finally, Experiment 4 investigated whether binocular vision was necessary for the contradirectional postural response to occur.

\section{GENERAL METHOD}

\section{Apparatus}

In Experiments 1-4, we recorded body responses in the lateral direction for different visual conditions. Since the main goal of the study was to characterize the direction of visually evoked postural responses, discrete unidirectional motion of a visual background was the chosen stimulus (Bronstein, 1986; Bronstein \& Buckwell, 1997). The visual background consisted of a $2 \times 3 \mathrm{~m}$ flat board $\left(67^{\circ} \times 90^{\circ}\right.$ of visual angle). Photoluminescent yellow-green stripes were used to create the picture of a house with a peaceful garden (Figure 2). Background displacement was achieved by mounting the flat board on a chassis with four pneumatic wheels (bogie) running on a linear track. The bogie was driven by a pair of linear induction motors that generated thrust against a reaction plate situated along the middle of the track. The background was moved at $150 \mathrm{~cm}$ from the subject's eyes along an axis parallel to the interaural axis in an otherwise dark room. It was moved $58 \mathrm{~cm}\left(21^{\circ}\right)$ leftward or rightward. A constant velocity of $6 \mathrm{~cm} / \mathrm{sec}$ was reached after approximately $1.25 \mathrm{sec}$ of acceleration onset and sustained for $8.5 \mathrm{sec}$ before the deceleration occurred. After each trial, the subjects were asked to close their eyes while the background was moved 


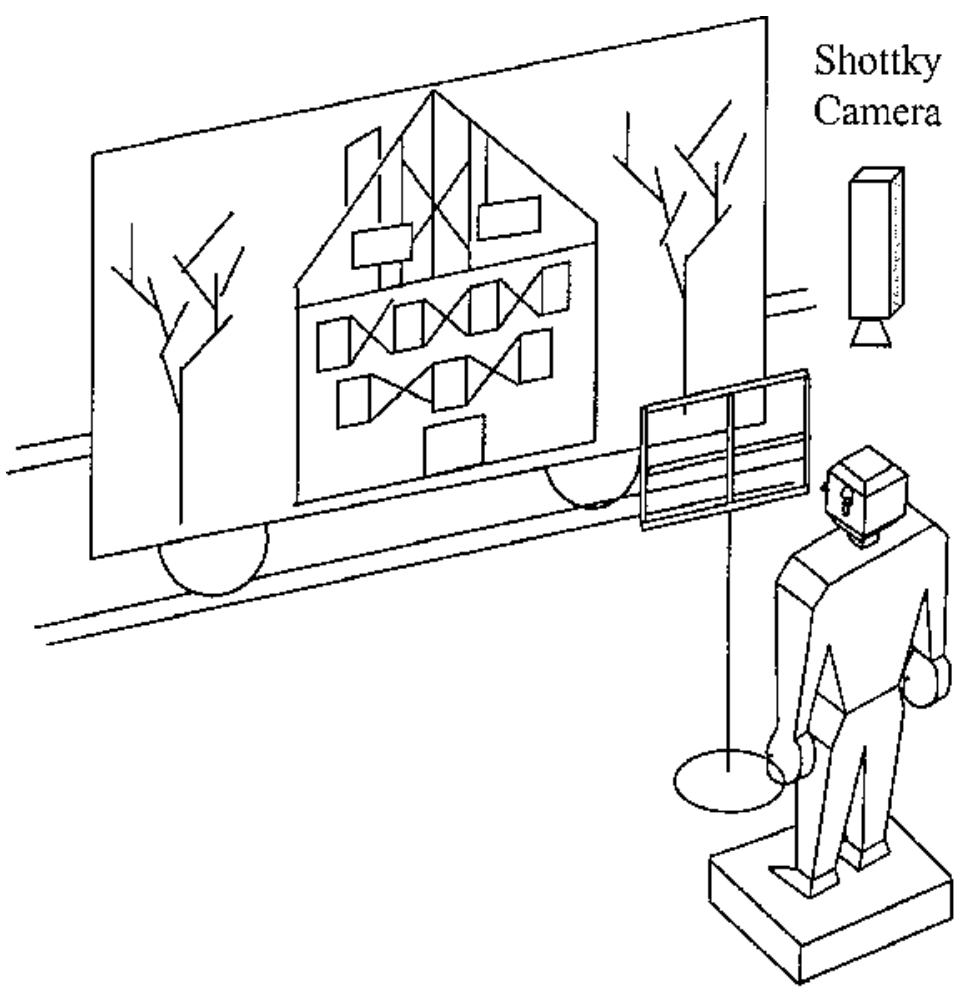

Force platform

Figure 2. Schematic diagram showing the experimental setup of Experiment 1.

back to its starting position and reilluminated with a lamp to keep a constant level of luminance.

\section{Procedure}

In all experiments, the subjects were instructed to "stand still and relaxed" with hands at their sides. They stood barefoot on a rigid foot support placed on top of a slab of foam rubber (height, $5 \mathrm{~cm}$; specific weight, $30 \mathrm{~g} / \mathrm{dm}^{3}$ ) resting on the sway platform. The aim was to increase the instability of the subjects so that any effect of visual condition on the subjects' sway could be more easily observed (Bles et al., 1980; Bronstein, 1986; Lee \& Lishman, 1975). Postural sway in the lateral direction was recorded using a force platform for center of pressure (COP) recordings. The subject wore a lightweight helmet carrying an infrared LED so that head displacements could be measured with a Shottky barrier photodetector camera (resolution of $0.1 \mathrm{~mm}$, linear up to $\pm 8 \mathrm{~cm}$ ) mounted $40 \mathrm{~cm}$ above the subject's scalp. Since the distance between the headmounted infrared light and the Shottky camera was maintained at $40 \mathrm{~cm}$, head-sway values were normalized for the subjects' heights (signal $\times$ mean height of group/individual's height). A computer was used to generate the command signal to the linear motors of the bogie and to acquire the output signals from the sway platform and the Shottky camera at a sampling rate of $125 \mathrm{~Hz}$. The sway signals were also continuously monitored on line on an oscilloscope. The experimenter familiarized himself with the spontaneous sway amplitude of each subject and delivered the stimuli at points of average or smaller than average sway amplitude.

Measurements taken from COP and head position signals included average response ( $X_{\mathrm{o}}$; Equation 1$)$, which measures body orientation relative to the prestimulus position value $\left(X_{\text {baseline }}\right)$ in the lateral direction. Average response indicates the directionally specific effect of any stimulus on head or COP position in space:

$$
X_{\mathrm{o}}=\frac{1}{N} \sum_{i=1}^{N}\left(x_{i}-X_{\text {baseline }}\right)
$$

where $N$ is the number of samples, $x_{i}$ is the COP/head position for the $i$ th sample, and $X_{\text {baseline }}$ is the average position (COP or head) during the $4 \mathrm{sec}$ preceding stimulus onset. It was calculated for each single trial during the $8.5 \mathrm{sec}$ of constant velocity and then averaged for each subject and visual condition. Stimulus onset was used to synchronize the recordings during averaging. This allowed the specific component of sway due to the visual stimuli to emerge. Since no differential effect between the two directions of motion was expected, responses to rightward and leftward stimuli were combined (after reversing all sway responses during leftward motion).

One-sample $t$ tests were used to obtain the significance of the effect of a given visual condition on average COP and head position values for each individual or for the group. Paired-samples $t$ tests were used for all comparisons of the mean values, based on individual averages. Spearman correlation coefficients were used to assess any relation between sway magnitudes for different conditions of visual fixation. A .05 significance level was adopted throughout.

\section{EXPERIMENT 1}

\section{Method}

Subjects. Twelve normal subjects with normal or corrected vision (normal visual acuity and stereopsis) gave their informed consent to participate in the experiment. The subjects ranged in age 
from 25 to 50 years old. All subjects were healthy, without musculoskeletal or neurological disorders.

Visual conditions. The three visual conditions to which each subject was exposed were the following: (1) looking straight ahead at the background that was the only visible display (background fixation) - the subjects were instructed to look straight ahead, binocularly, at the house in the background without following it when it moved; (2) fixating a cross $(1 \times 1 \mathrm{~cm})$ placed at the center of a purpose-built earth-fixed luminescent window frame $(30 \times 24 \mathrm{~cm})$, at a distance of $50 \mathrm{~cm}$ from the subject's eyes (Figure 2; foreground fixation); and (3) looking straight ahead at the background through the earth-fixed window frame $50 \mathrm{~cm}$ away (background fixation through the window). Each visual condition consisted of 24 trials8 with motion to the right, 8 to the left, and 8 with stationary background (shams) - equally divided into two groups of 12 pseudorandomized stimuli. The first three blocks (one per visual condition), followed by the second three blocks, were presented in a Latin-square design. A rest of 15 min was given to the subjects between the two test sessions.

\section{Results and Discussion}

Figure 3 shows a typical sample record of head and COP displacements during background and foreground fixation conditions. Head and COP displacements followed a similar trend during the whole recording in the three different visual conditions. Values for the COP and the head displacements are reported in Table 1.

When the subjects were looking straight at the background as shown in the sample record in Figure 3 (background fixation condition, left panel), an initial postural readjustment was observed in the direction of motion with a latency of approximately $800 \mathrm{msec}$. This initial response was followed by a resetting, which brought the COP and the head toward their initial baseline positions. The postural resetting was then followed by an increasing displacement of the COP and the head in the direction of background motion until the end of the stimulus, as shown in the sample record in Figure 3. When the movement of the visual background stopped, a postural correction bringing the COP and the head toward their initial baselines was observed. By synchronizing stimulus onset, the initial postural response can be clearly identified in the average from individual data (Figure $4, n=12$ ). The mean slopes of the initial postural response computed for each individual subject were $6.7 \mathrm{~mm} / \mathrm{sec}$ for the COP and $4.1 \mathrm{~mm} / \mathrm{sec}$ for the head. As shown in Figure 4, the initial codirectional postural response during background fixation was followed by a postural correction in the op-

\section{Background fixation}

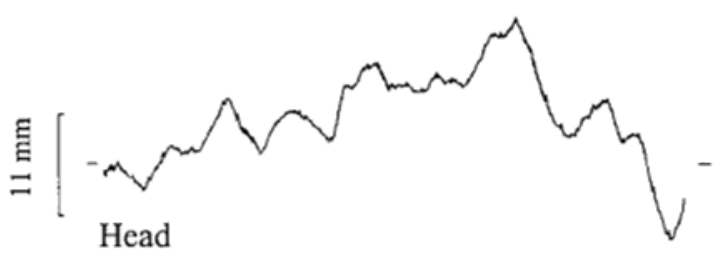

Head

\section{Foreground fixation}

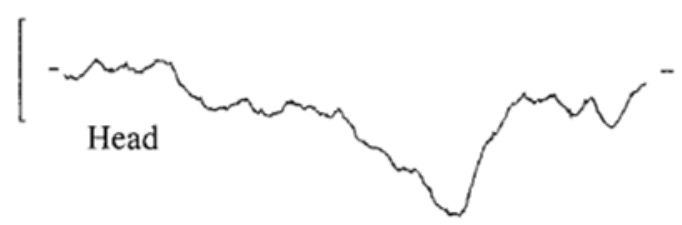

Background motion
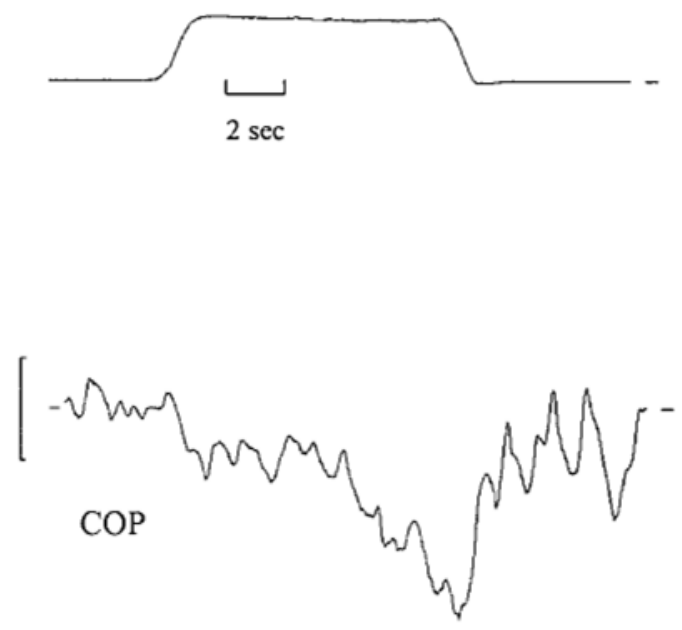

Figure 3. A sample record of a postural response induced by the moving background in background fixation condition (left panel) and in foreground fixation condition (right panel). Upward deflections indicate deviation in the direction of stimulus motion. 
Table 1

Average Position (Mean and Standard Deviation; in Millimeters) of the COP and the Head According to the Visual Condition in Experiment 1

\begin{tabular}{|c|c|c|c|c|c|c|c|c|}
\hline & \multicolumn{8}{|c|}{ Condition } \\
\hline & \multicolumn{2}{|c|}{ Background } & \multicolumn{2}{|c|}{ Foreground } & \multicolumn{2}{|c|}{$\begin{array}{l}\text { Background } \\
\text { Window }\end{array}$} & \multicolumn{2}{|c|}{ Sham } \\
\hline & $M$ & $S D$ & $M$ & $S D$ & $M$ & $S D$ & $M$ & $S D$ \\
\hline Head sway & 3.41 & 4.01 & -5.38 & 2.70 & -2.42 & 3.07 & 0.27 & 0.61 \\
\hline COP & 2.96 & 2.67 & -4.01 & 1.63 & -1.27 & 1.97 & 0.26 & 0.74 \\
\hline
\end{tabular}

posite direction, bringing the $\mathrm{COP}$ (and the head) to baseline levels. The position of both the COP and the head at this point, computed for each individual subject, did not differ from the baseline level [COP, $t(11)=0.1, p=.94$; head, $t(11)=0.98, p=.34$ ]. One-sample $t$ tests, based on the average response of each subject during the $8.5 \mathrm{sec}$ of constant background velocity, confirmed the significance of deviation in the direction of background motion [COP, $t(11)=3.8, p<.01$; head, $t(11)=2.9, p<.05]$. Seventysix percent of single trials were found to be in the direction of stimulus motion. Three of the 12 subjects tested showed no statistically significant postural adjustment.

During fixation of the foreground target (foreground fixation condition), a displacement of the COP and of the head was observed in the opposite direction of background motion as shown in the sample recording presented in Figure 3 (right panel). The mean latency of the postural response was around $850 \mathrm{msec}$ (Figure 4) and was not significantly different from that observed in the condition of background fixation [COP, $t(11)=1.9, p=$ .08 ; head, $t(11)=0.9, p=.40]$. The slopes of the initial postural response were $-3.1 \mathrm{~mm} / \mathrm{sec}$ and $-2.1 \mathrm{~mm} / \mathrm{sec}$ for COP and head displacements, respectively, and were significantly less steep than the slopes of the initial postural response observed in the condition of background fixation [COP, $t(11)=3.8, p<.01$; head, $t(11)=2.8$, $p<.05]$. The initial postural displacement in the opposite direction to that of the visual stimulus was not followed by a sharp postural correction toward the baseline (Figure 4). Instead, the subjects continued to lean in the opposite direction of stimulus motion until it stopped. The average peak displacements in the opposite direction of motion were $6 \mathrm{~mm}$ and $8 \mathrm{~mm}$ for the COP and the head, respectively. One-sample $t$-test analysis confirmed that the displacements of both the COP and the head in the opposite direction of background motion were statistically significant [COP, $t(11)=9.4, p<.01$; head, $t(11)=6.9, p<.01]$. The postural readjustments in the opposite direction of motion were significant in all subjects except one. Eighty-nine percent of responses to the single trials were in the opposite direction of stimulus motion. The COP and head displacements recorded during foreground and background fixation were negatively correlated (COP, $r=-.62, p<.05$; head, $r=-.60, p<$ $.05)$. Therefore, the more a subject deviated in the direc- tion of motion during background fixation, the more this subject deviated in the opposite direction during foreground fixation (Figure 5).

During the condition of background fixation through the window, a slight tendency to lean in the opposite direction of background motion was observed (Figure 4). This effect was statistically significant when individual averages were considered [COP, $t(11)=2.2, p<.05$; head, $t(11)=2.7, p<.05$ ] but was not consistent since it was significant in only 4 of the 12 subjects tested. Sixty percent of responses were in the opposite direction of stimulus motion. The amplitude of the postural displacement observed in this visual condition was statistically different from that observed in background [COP, $t(11)=$ $3.7, p<.01$; head, $t(11)=3.1, p<.01]$ and foreground [COP, $t(11)=3.6, p<.01$; head, $t(11)=4.3, p<.01]$ conditions. No significant correlations with the other two conditions were observed for either the COP or the head recordings.

No difference in average response was found between the three sham conditions (trials for each visual fixation condition with stationary background). All trials were combined to obtain a single average per subject, and no consistent body movement to the right or left appeared when the background was stationary (Figure 4).

The slower (i.e., less steep) initial postural displacement observed during foreground fixation, relative to that during background fixation, would be consistent with the visual flow experienced by a subject swaying in a stable environment. A spontaneous displacement of the head at $1 \mathrm{~cm} / \mathrm{sec}$ when fixating a stationary background placed at $150 \mathrm{~cm}$ from the eyes produces a retinal image motion $(0.39 \% \mathrm{sec})$. This is approximately half the amount of retinal image motion of the background that subjects experience when fixating a foreground object placed at $50 \mathrm{~cm}$ from the eyes $(0.76 \% \mathrm{sec})$ (see Guerraz, Sakellari, Burchill, $\&$ Bronstein, 2000). Thus, to produce a similar retinal image motion of the background in these two visual conditions, the velocity of the visual background should be twice as fast in the foreground fixation condition as in the background fixation condition.

The absence of visually induced body sway in response to background motion in the condition of background fixation in 3 of the 12 subjects tested in Experiment 1 reflects the important interindividual differences consis- 

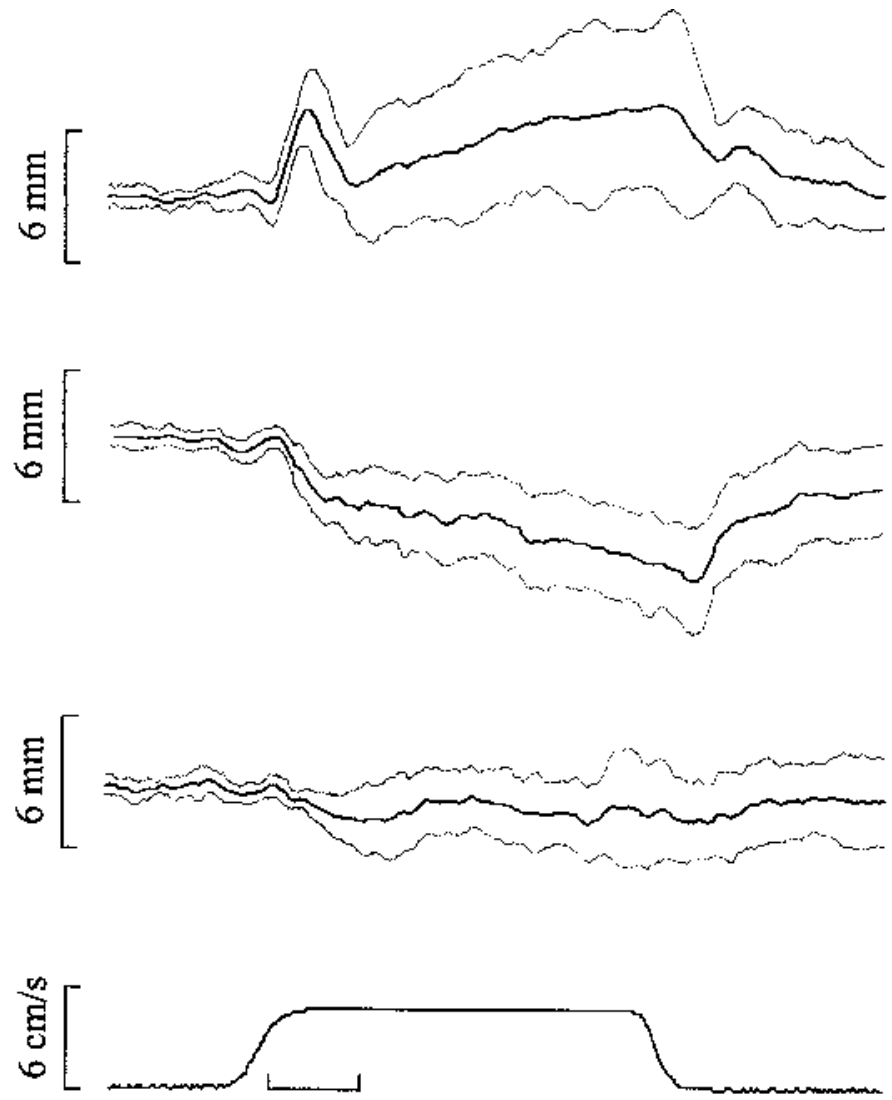

$2 \sec$
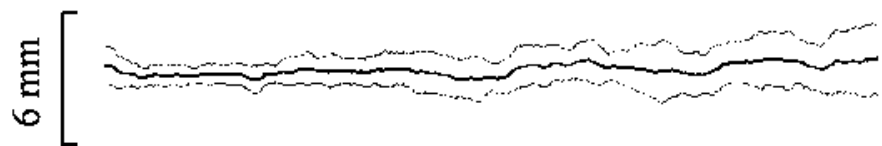

Background fixation

\section{Foreground}

fixation

\section{Background motion}

Figure 4. COP displacements evoked by a moving visual background during the background condition, the foreground condition, and the condition of fixation through the window (foreground). COP displacement with a stationary background is also reported at the bottom of the figure. The average positions and standard deviations of the COP displacements in the 12 normal subjects are shown. Upward deflections indicate a displacement in the direction of stimulus motion; downward deflections indicate a displacement in the opposite direction of motion.

tently reported in the field of visual control of balance (Crémieux \& Mesure, 1994; Isableu, Ohlmann, Crémieux, \& Amblard, 1998; Lacour et al., 1997; Lestienne et al., 1977; Masson, Mestre, \& Pailhous, 1995; Warren et al., 1996). For instance, Lestienne et al. (1977) reported that $20 \%$ of subjects tested with linear motion of the visual scene did not show any postural readjustment. Although the reason is not well understood (see Isableu et al., 1998), a postural response could be induced in some of these nonresponsive subjects when they were asked to do a mental arithmetic task at the same time as the pos- tural task (Lestienne et al., 1977), indicating that attentional mechanisms could affect the postural response. The 3 above-mentioned subjects showing no visually induced body sway in response to background motion in Experiment 1 were not included in subsequent experiments.

In summary, we replicated the observation of Bronstein and Buckwell (1997) showing that by fixating a stationary foreground placed between the subject and a moving background, the postural response induced by motion of the background is reversed in direction. In ad- 


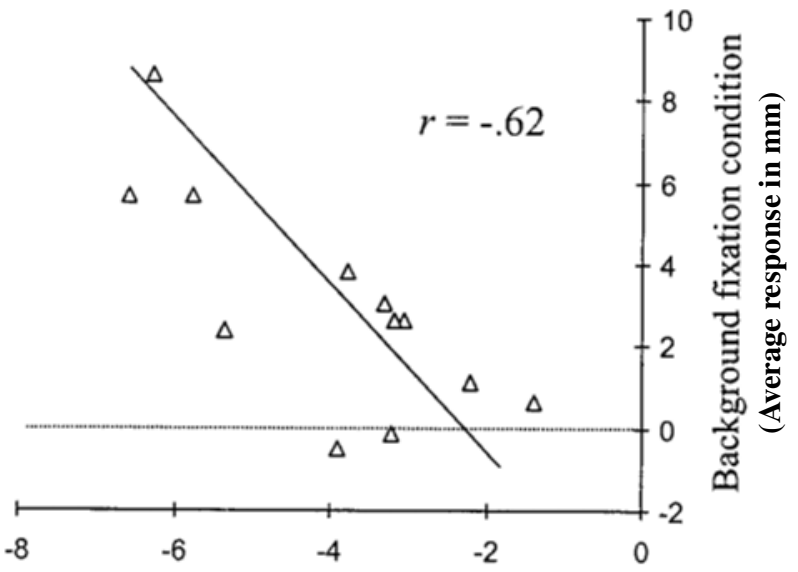

Foreground fixation condition

(Average response in $\mathbf{~ m m}$ )

Figure 5. Amplitude of the average response of the COP (in millimeters) for each subject in the condition of direct background fixation (vertical axis) plotted against the average response of the COP in the condition of foreground fixation (horizontal axis).

dition, we showed that this contradirectional postural response was not transient: It was sustained during the 11sec stimulus.

\section{EXPERIMENT 2}

The aim of Experiment 2 was to investigate the respective roles of motion parallax and induced movement in the contradirectional postural response observed in Experiment 1 . Induced movement is most effectively elicited when induced and inducing stimuli are as close as possible in all three dimensions of space (adjacency principle: Gogel \& Koslow, 1972; Gogel \& MacCracken, 1979; Reinhardt-Rutland, 1988), whereas motion parallax increases with increasing distance between two objects. As a consequence, if the contradirectional sway observed in foreground fixation condition (Experiment 1) was elicited by induced movement of the foreground, it should be even greater and more consistent when the earth-fixed foreground is coplanar with the moving background than when the foreground and background are at $100 \mathrm{~cm}$ from each other.

\section{Method}

Subjects. Eight normal subjects with normal or corrected vision gave their informed consent to participate in the experiment. The subjects ranged in age from 24 to 50 years old. Three of these subjects had participated in Experiment 1.

Visual conditions. Parameters of background motion were the same as those in Experiment 1. The subjects were exposed to three visual conditions: (1) looking straight ahead at the background as in Experiment 1 (background fixation condition); (2) fixating a laser point projected on a small earth-fixed black dot $(1 \mathrm{~cm}$ of diameter) taped on a piece of Perspex and adjusted at eye level-the fixation point was at $50 \mathrm{~cm}$ from the subject's eyes and at $100 \mathrm{~cm}$ from the background (foreground condition); and (3) fixating an earth-fixed laser point projected directly on the background (coplanar condition).

Each condition consisted of 21 trials -7 to the right, 7 to the left, and 7 sham (no motion) stimuli-divided into two groups of 10 or 11 pseudo-randomized stimuli. The first three blocks (one per condition) followed by the second three blocks were presented in a Latin-square design. The subjects were asked at the end of each block for the two visual conditions with laser-point fixation to tell the experimenter whether or not and how the laser point appeared to move with respect to the background.

\section{Results and Discussion}

In the coplanar condition, 7 subjects reported seeing either systematically or from time to time the earth-fixed laser point moving in the opposite direction of background motion. In the foreground condition, only 1 subject reported seeing the laser point moving. Values for the COP and the head displacements are reported in Table 2.

When the subjects were looking straight at the background (background condition), the motion stimulus induced a body readjustment in the direction of motion (Figure 6 , top trace). The pattern of sway was similar to that observed in Experiment 1. One-sample $t$-test analysis confirmed that this deviation in the direction of motion was statistically significant [COP, $t(7)=5.02, p<.01$; head, $t(7)=3.3, p=.01]$. This deviation was not significant in 2 of the 8 subjects tested.

In the foreground fixation condition, the linear motion of the background induced a clear postural readjustment in the opposite direction of motion (Figure 6, middle trace) as in Experiment 1. One-sample $t$-test analysis confirmed

Table 2

Average Response (Mean and Standard Deviation; in Millimeters) of the COP and the Head According to the Visual Condition in Experiment 2

\begin{tabular}{|c|c|c|c|c|c|c|c|c|}
\hline & \multicolumn{8}{|c|}{ Condition } \\
\hline & \multicolumn{2}{|c|}{ Background } & \multicolumn{2}{|c|}{$\begin{array}{c}\text { Motion } \\
\text { Parallax } \\
\end{array}$} & \multicolumn{2}{|c|}{$\begin{array}{c}\text { Induced } \\
\text { Movement }\end{array}$} & \multicolumn{2}{|c|}{ Sham } \\
\hline & $M$ & $S D$ & $M$ & $S D$ & $M$ & $S D$ & $M$ & $S D$ \\
\hline Head & 3.52 & 3.03 & -3.68 & 2.27 & 0.68 & 2.31 & 0.64 & 1.04 \\
\hline $\mathrm{COP}$ & 3.1 & 1.7 & -2.8 & 1.53 & 1.08 & 1.7 & 0.42 & 0.6 \\
\hline
\end{tabular}

Note-A positive value indicates a deviation in the direction of motion, whereas a negative value indicates a deviation in the opposite direction of motion. 


\section{Background condition}

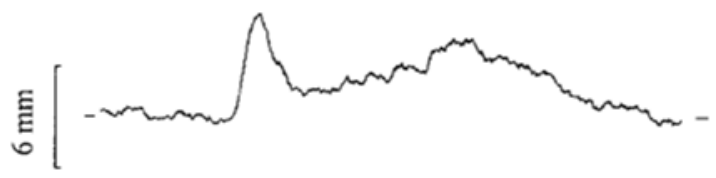

Foreground condition

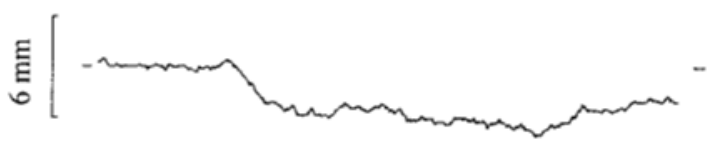

\section{Coplanar condition}

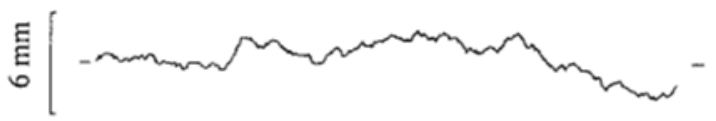

Background motion

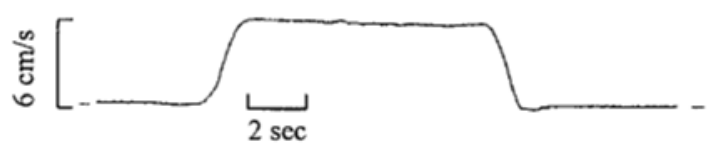

Figure 6. Postural readjustments evoked by a moving visual background during conditions of background fixation (top), foreground fixation (middle), and coplanar fixation (bottom). The averages of the COP in the 8 normal subjects are shown. Upward deflections indicate deviation in the direction of stimulus motion.

a significant displacement of the body in the opposite direction of stimulus motion $[\mathrm{COP}, t(7)=5.15, p<.01$; head, $t(7)=3.2, p<.05]$. This displacement was not significant in 2 of the 8 subjects tested.

In the coplanar condition, a small sway in the direction of background motion was observed (Figure 6, bottom trace) but was not statistically significant [COP, $t(7)=$ $1.7, p=.12$; head, $t(7)=0.8, p=.43$ ]. A significant deviation in the direction of background motion was observed in only 2 of the 8 subjects; $59 \%$ of the total single responses were in the direction of motion, and $41 \%$ were in the opposite direction. Postural readjustments observed in the coplanar condition were significantly smaller than those observed in the background condition [COP, $t(7)=$ $3.2, p<.05$; head, $t(7)=2.5, p<.05]$ and the foreground condition $[\mathrm{COP}, t(7)=5.3, p<.01$; head, $t(7)=4.3, p<$ $.01]$. The average body position observed when the background was stationary (sham trials) was not different from zero, whatever the viewing condition $(p>.05)$.

In summary, a consistent contradirectional postural response was elicited only in the presence of depth be- tween the fixation point and the moving background (foreground condition) but not when the fixation point and the moving background were coplanar. The results of Experiment 2 suggest that, in the lateral direction, motion parallax is a more relevant cue to elicit a contradirectional sway response than is induced motion.

\section{EXPERIMENT 3}

The aim of Experiment 3 was to examine whether the possibility that the contradirectional postural response described in Experiments 1 and 2 was due to ocular con-

\section{Background condition}

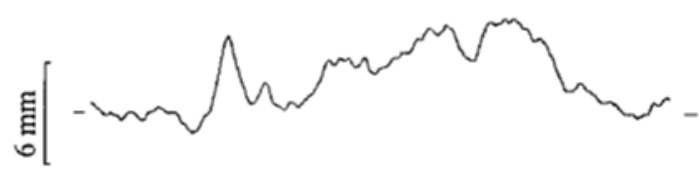

\section{Earth-fixed foreground condition}

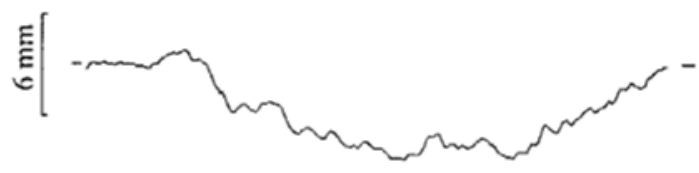

Head-fixed foreground condition
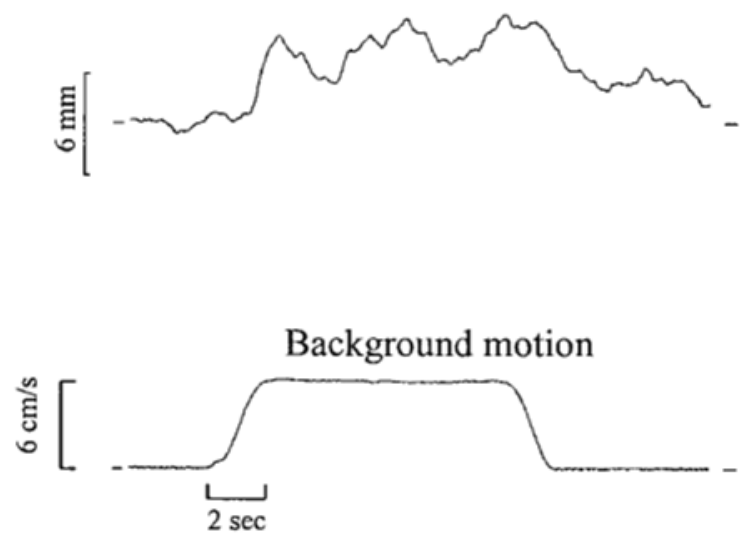

Figure 7. Postural readjustments evoked by a moving visual background while the subject was looking straight ahead at the background (top trace), fixating an earth-fixed foreground consisting of an LED (middle trace), and fixating a head-fixed foreground (bottom trace). The averages of the COP in the 7 normal subjects are shown. Upward deflections indicate deviation in the direction of stimulus motion. 
vergence (in front of the background) rather than to motion parallax. Convergence on a fixation point in the foreground produces a defocused double image of the visual background behind. The defocusing reduces spatial frequency corresponding to a reduction in visual acuity, which has been found to play a significant role in the regulation of lateral sway in a stable environment (Paulus, Straube, \& Brandt, 1984).

\section{Method}

Subjects. Seven subjects with normal or corrected vision gave their informed consent to participate in this experiment. The subjects ranged in age from 21 to 52 years old. Three of the 7 subjects had participated in either Experiment 1 or Experiment 2. One subject had to stop in the middle of the experiment because of nausea. Since this subject took part in the first three blocks of the experiment (one block per visual condition), his data were included in the analysis of the experiment.

Visual conditions. Parameters of background motion were the same as those in Experiment 1. The subjects were exposed to three visual conditions: (1) looking straight ahead at the background, as in Experiments 1 and 2 (background condition); (2) fixating an LED placed on a piece of Perspex adjusted to eye level—the fixation point was at $40 \mathrm{~cm}$ from the subject's eyes and at $110 \mathrm{~cm}$ from the background (earth-fixed foreground condition); and (3) fixating an LED at $40 \mathrm{~cm}$ from the subject's eyes, attached to the helmet with a rod (head-fixed foreground condition). For both head-fixed and earthfixed foreground, convergence and accommodation remained the same, and the background remained out of focus. Unlike in the condition of earth-fixed foreground, when the fixation point is coupled with head movement, the opposite motion between the nearer object and the farther one as shown in Figure 1 does not hold: When moving the head to one side, the background will be perceived as moving in the opposite direction of head motion as in the condition of direct background fixation. Then, as for the condition of direct background fixation, a sway codirectional to background motion was expected.

Each condition consisted of 12 stimuli- 4 to the right, 4 to the left, and 4 shams (no motion)—divided into two groups of 6 pseudorandomized stimuli. The first three blocks (one per condition) followed by the second three blocks were counterbalanced. Observation of the different visual scenes was binocular, as in Experiments 1 and 2. A rest of 15 min was given to the subjects between the two test sessions.

\section{Results and Discussion}

Figure 7 shows the mean COP displacement obtained with the 7 subjects for each visual condition. Similar results were observed for head displacement (Table 3).

When the subjects were looking straight at the background (background condition), the linear motion induced a body readjustment in the direction of motion (Figure 7, top trace). As in Experiments 1 and 2, the fixation of an earth-fixed object (LED) in front of the moving background induced a clear body readjustment in the opposite direction of motion (Figure 7, middle trace).

With the head-fixed LED, a body readjustment in the direction of background motion was elicited. As shown in Figure 7 (bottom trace), the pattern of sway was similar to the sway observed when the subjects looked straight at the background. The displacement of the COP was even greater in the head-fixed condition than in the background condition [COP, $t(6)=2.9, p<.05$; head, $t(6)=$ $2.45, p=.05]$.

In summary, it was found that when the fixated foreground was coupled with head movement, a codirectional sway to background motion was elicited. Its amplitude appeared even greater and more consistent than the codirectional sway observed with direct background fixation. Hence, convergence in front of the moving visual surround is not sufficient to elicit a contradirectional postural response.

\section{EXPERIMENT 4}

The aim of Experiment 4 was to compare the condition of foreground fixation when viewed monocularly and binocularly. Since motion parallax is primarily monocular, a contradirectional postural response should be observed with both monocular and binocular vision.

\section{Method}

Subjects. Nine subjects with normal or corrected vision gave their informed consent to participate in this experiment. The subjects ranged in age from 15 to 52 years old. Four of the 9 subjects had participated in one of the first three experiments. Since the purpose of this experiment was to compare the effect of binocular versus monocular viewing on the reversed response, 1 subject who exhibited a systematic codirectional sway in the earth-fixed foreground condition was excluded from data analysis.

Visual conditions. The motion of the background condition and the earth-fixed foreground fixation condition was the same as in Experiment 1 (Figure 2). The subjects were fixating the foreground binocularly or monocularly with their dominant eye, the second eye being covered by a patch. Three subjects had a left dominant eye, and 5 had a right dominant eye. Each condition consisted of 15 stimuli-5 to the right, 5 to the left, and 5 shams (no motion)—divided into two groups of 7 or 8 pseudo-randomized stimuli. The first two blocks (one per condition) followed by the second two blocks were counterbalanced. A rest of 15 min was given to the subjects between the two test sessions.

Table 3

Average Response (Mean and Standard Deviation; in Millimeters) of the COP and the Head According to the Visual Conditions in Experiment 3

\begin{tabular}{|c|c|c|c|c|c|c|c|c|}
\hline & \multicolumn{8}{|c|}{ Condition } \\
\hline & \multicolumn{2}{|c|}{ Background } & \multicolumn{2}{|c|}{$\begin{array}{l}\text { Head-Fixed } \\
\text { Foreground } \\
\end{array}$} & \multicolumn{2}{|c|}{$\begin{array}{l}\text { Earth-Fixed } \\
\text { Foreground }\end{array}$} & \multicolumn{2}{|c|}{ Sham } \\
\hline & $M$ & $S D$ & $M$ & $S D$ & $M$ & $S D$ & $M$ & $S D$ \\
\hline Head & 3.64 & 4.02 & 5.96 & 2.2 & -6.5 & 6.3 & 1.2 & \\
\hline $\mathrm{COP}$ & 2.66 & 2.5 & 4.49 & 1.9 & -3.66 & 4.03 & 0.5 & 0.8 \\
\hline
\end{tabular}

Note-A positive value indicates a deviation in the direction of motion, whereas a negative value indicates a deviation in the opposite direction of motion. 
Table 4

Average Response (Mean and Standard Deviation; in Millimeters) of the COP and the Head in Foreground Condition With Monocular and Binocular Fixation in Experiment 4

\begin{tabular}{|c|c|c|c|c|c|c|}
\hline & \multicolumn{6}{|c|}{ Condition } \\
\hline & \multicolumn{2}{|c|}{$\begin{array}{c}\text { Binocular Fixation } \\
\text { (Moving Background) }\end{array}$} & \multicolumn{2}{|c|}{$\begin{array}{c}\text { Monocular Fixation } \\
\text { (Moving Background) }\end{array}$} & \multicolumn{2}{|c|}{ Sham } \\
\hline & $M$ & $S D$ & $M$ & $S D$ & $M$ & $S D$ \\
\hline Head sway & -6.4 & 2.9 & -5.4 & 3.1 & -0.2 & 0.9 \\
\hline $\mathrm{COP}$ & -4.9 & 2.1 & -3.8 & 2.4 & -0.1 & 0.8 \\
\hline
\end{tabular}

Note-A positive value indicates a deviation in the direction of motion, whereas a negative value indicates a deviation in the opposite direction of motion.

\section{Results and Discussion}

For both viewing conditions, the linear visual motion induced a clear displacement in the opposite direction of background motion, as in Experiments 1-3 (Table 4). The amplitude of the contradirectional sway response was slightly but significantly larger during binocular fixation than during monocular fixation for the $\operatorname{COP}[t(8)=$ $3.4, p<.05]$. Similar results were observed for head position, except that the difference between the two viewing conditions did not reach significance $[t(8)=1.6, p=.15]$. As expected, when the background remained stationary (sham condition), no postural displacement was observed in either viewing condition.

The results of Experiment 4 showed that the contradirectional postural response during foreground fixation was observed during both monocular and binocular conditions. This indicates that the contradirectional response is, in the main, monocularly mediated. The trend for larger binocular responses than monocular responses is in agreement with previous studies on spontaneous body sway showing improved body stability during binocular fixation relative to that during monocular fixation (Fox, 1990; Jones \& Lee, 1981; Paulus et al., 1984; Stoffregen, Smart, Bardy, \& Pagulayan, 1999).

\section{GENERAL DISCUSSION}

In the present study, we attempted to further define the significant factors determining the contradirectional postural response previously reported by Bronstein and Buckwell (1997) when a stationary fixated foreground was placed between a moving background and a standing subject. The results of Experiments 1-4 show that this contradirectional postural response was not just a transient response but could be sustained at least for the $11 \mathrm{sec}$ of background motion. The results of these experiments also provide converging evidence that this contradirectional sway elicited by earth-fixed foreground fixation against a moving background was due to motion parallax. The key arguments are the following: (1) The presence of an earth-fixed foreground target reversed the postural response induced by the moving background when the direction of displacement of the two objects on the retina mimics what an observer would experience during self-motion in a stable environment (foreground fixation). When the direction of displacement of the two objects on the retina are inconsistent with what a moving observer would experience, the postural response was found to be inconsistent (fixation through the window condition). (2) The effect is not mediated by induced movement. Although most of the subjects reported an induced movement illusion when the fixation point was coplanar to the moving background, no systematic contradirectional postural response was observed (Experiment 2). (3) The contradirectional postural response is not caused by ocular convergence/accommodation on a foreground target against a defocused moving background, since codirectional sway was elicited with a head-fixed target (Experiment 3 ). Finally, this contradirectional postural response could be observed monocularly and binocularly (Experiment 4). Thus, the necessary condition for reversed sway seems to be a differential motion of foreground with respect to the background similar to that which would be provoked by self-motion of the observer.

As suggested recently, control of stance is not independent of other suprapostural behavioral tasks (Riccio \& Stoffregen, 1988; Stoffregen et al., 1999). For instance, in order to shoot a target with a gun, one requires minimizing sway in order to aim successfully. Thus, the fixation task can significantly affect the control of stance. The data presented here would suggest that one of the ways in which this could come about is by using parallax cues emerging as a result of the new fixation task. Disentangling suprapostural fixation task from parallaxmediated effects can, however, prove difficult. As mentioned above, the visual flow that a subject would experience in a 3-D environment is modulated by the fixation point. As shown in Figure 1, when a moving subject is fixating a given object, nearer objects move on the retina in the opposite direction of self-motion, whereas far objects move in the direction of motion. Thus, one could argue that the reversal of body sway observed in the foreground conditions of Experiments 1-4 and previously (Bronstein \& Buckwell, 1997) could be the consequence of the fixation task per se rather than motion parallax. Data from Experiment 1 and recent research (Guerraz et al., 2000), however, do not support this hypothesis. In Experiment 1, the fixation task in the condition of back- 
ground fixation and in the condition of background fixation through the window was the same: The subjects look straight at the background. If the fixation task was the relevant factor controlling postural readjustment, a similar postural response should be observed in these two conditions. On the contrary, the postural readjustment triggered by motion of the background was entirely different. When fixating the background with no objects in the foreground, the subjects showed the well-established codirectional postural response (Lee \& Lishman, 1975; Lestienne et al., 1977). When subjects fixate the background through the foreground window, the response is significantly smaller in amplitude, inconsistent, and with a contradirectional preference, as in shown in the present study and a previous study (Bronstein \& Buckwell, 1997). In addition, we have recently shown that motion parallax plays a specific role in the control of spontaneous body sway in a stable 3-D environment (Guerraz et al., 2000 ), in which the effect of motion parallax was observed when depth information was manipulated and the fixation task was always kept constant. In those experiments, subjects always fixated an LED at $50 \mathrm{~cm}$ from the eye while motion parallax was increased by placing additional LED targets at different distances from the fixated LED. The results showed that the low-frequency components of sway $(<0.5 \mathrm{~Hz})$ were significantly reduced with increasing distance between LEDs. Thus, although the fixation task can be an important factor affecting postural responses, it can explain neither the effect of depth information observed on spontaneous body sway (Guerraz et al., 2000) nor that observed on visually induced body sway (Experiments $1-4$ reported here).

The retinal periphery has been considered to be dominant for self-motion perception and the control of posture (Brandt, Wist, \& Dichgans, 1975; Dichgans \& Brandt, 1978). However, Andersen and Dyre (1989) found that visually induced body sway could be elicited when stimulation was restricted to a small area of the central visual field $\left(15^{\circ}\right)$. Moreover, optical information for the control of posture also appears to be a function of the geometric structure of the light rays that form the optical flow field (Gielen \& van Asten, 1990; Masson et al., 1995; Stoffregen, 1985, 1986). Stoffregen (1985) demonstrated that the retinal periphery itself shows no particular facility for detecting posturally relevant information if the visual flow is radial as opposed to lamellar. The central versus peripheral distinction also applied for near and far vision. Delorme and Martin (1986) found that both the retinal periphery and the depth periphery played important roles in the visually induced body sway. They showed that forward and backward movement of the scene limited to the foreground produced little postural sway in the anteroposterior direction relative to motion located in the background. In the foreground fixation condition in Experiments 1-4, the "central object," in terms of both retinal area and depth, reversed the driving effect of the "peripheral stimulus." Our results suggest that the am- plitude of postural readjustment in both background and foreground fixation conditions was under the control of the moving background, as shown by the similar amplitude of both the codirectional and the contradirectional sway and by the significant correlation observed between these conditions (Figure 5). However, while the amplitude of postural readjustment appeared to be under the control of the moving background, the spatial relation between the background, the foreground, and the observer's fixation point determined the direction of the postural readjustment. These results provide more evidence that the control of stance is dependent not only on the retinal location of objects and on the geometric structure of the visual flow but also on the observer's fixation point in a 3-D environment. Then, when present in the visual environment, motion parallax is a powerful cue to specify the direction of sway in the frontal plane.

As mentioned above, it remained possible that the induced movement of the foreground rather than motion parallax was the relevant cue to reverse the postural sway to background motion. The purpose of Experiment 2 was to test this alternative explanation. Although most subjects reported the illusory motion of the fixation point when coplanar to the moving background, no consistent direction-specific postural response was observed. Unlike motion parallax, induced movement can exist in the absence of an observer's own motion. Our results do not exclude the possibility that induced movement, in some trials or circumstances, can induce contradirectional postural response, as proposed by van Asten et al. (1988) for the sagittal plane (i.e., induced motion in depth), but its contribution in the lateral direction appears to be less important than that of motion parallax.

The absence of direction-specific postural response was also observed in the condition of background fixation through the window (Experiment 1). As in the coplanar condition (Experiment 2), the relative movement of the two objects in the condition of background fixation through the window does not signal any real selfbody movement the subject could experience in the condition of stationary background. However, motion parallax in this condition could signal passive motion (e.g., a subject seated in a car or in a bus). As shown in Figure 1, when a subject is moving his/her head while looking beyond a near object, the image of the fixated point remains stable on the retina while the image of the near object moves in the opposite direction of head motion. To mimic more realistic self motion when the subject is looking at a far object, as in the condition of background fixation through the window, there should be movement of the near object. These findings suggest that in order to induce consistent and directionally specific responses, the relative motion of objects within the foreground and the background must reflect what a moving observer would experience in a stable 3-D environment. Note that the retinal displacement of the moving background $\left(21^{\circ}\right)$ in Experiments 1-4 was larger than what a subject would experience during spontaneous sway (Bles et al., 1980). 
However, it has been reported that stimulus amplitude is not a relevant parameter for visuopostural responses (Lestienne et al., 1977; Masson et al., 1995; van Asten et al., 1988).

In conclusion, the results of the present experiments clearly demonstrate that motion parallax can be a significant factor in postural orientation in standing subjects, as it has been found earlier in walking subjects (Bardy et al., 1996; Warren et al., 1996). Despite strong biomechanical differences, the visual control of standing and walking in a 3-D environment appears to be regulated by similar visual cues among which motion parallax is of particular importance.

\section{REFERENCES}

Andersen, G. J., \& Dyre, B. P. (1989). Spatial orientation from optic flow in the central visual field. Perception \& Psychophysics, 45, 453-458.

Bardy, B. G., Warren, W. H., \& KAY, B. A. (1996). Motion parallax is used to control postural sway during walking. Experimental Brain Research, 111, 271-282.

Bles, W., Kapteyn, T. S., Brandt, T., \& Arnold, F. (1980). The mechanism of physiological height vertigo. Acta Otolaryngologica, 89, 534-540.

Brandt, T., Wist, E. R., \& Dichgans, J. (1975). Foreground and background in dynamic spatial orientation. Perception \& Psychophysics, 17, 497-503.

Bronstein, A. M. (1986). Suppression of visually evoked postural responses. Experimental Brain Research, 63, 655-658.

Bronstein, A. M., \& BuCKWELl, D. (1997). Automatic control of postural sway by visual motion parallax. Experimental Brain Research, 113, 243-248.

Collewijn, H., Curio, G., \& Grüsser, O. J. (1982). Spatially selective visual attention and generation of eye pursuit movements. Human Neurobiology, 1, 123-139.

Crémieux, J., \& Mesure, S. (1994). Differential sensitivity to static visual cues in the control of postural equilibrium in man. Perceptual \& Motor Skills, 78, 67-74.

Delorme, A., \& Martin, C. (1986). Roles of retinal periphery and depth periphery in linear vection and visual control of standing in humans. Canadian Journal of Psychology, 40, 176-187.

Dichgans, J., \& BRANDT, T. (1978). Visual-vestibular interaction: Effects on self-motion perception and postural control. In R. Held, H. Leibowitz, \& H. Teuber (Eds.), Handbook of sensory physiology (Vol. 8, pp. 755-804). New York: Springer-Verlag.

Dichgans, J., Held, R., Young, L. R., \& Brandt, T. (1972). Moving visual scenes influence the apparent direction of gravity. Science, 178, 1217-1219.

Dijkstra, T. M., Schöner, G., \& Gielen, C. C. (1994). Temporal stability of the action-perception cycle for postural control in a moving visual environment. Experimental Brain Research, 97, 477-486.

Fluckiger, M., \& Baumberger, B. (1988). The perception of an optical flow projected on the ground surface. Perception, 17, 633-645.

Fox, C. R. (1990). Some visual influences on human postural equilibrium: Binocular versus monocular fixation. Perception \& Psychophysics, 47, 409-422.

Gielen, C. C., \& van Asten, W. N. (1990). Postural responses to sim- ulated moving environments are not invariant for the direction of gaze. Experimental Brain Research, 79, 167-174.

Gogel, W. C., \& Koslow, M. (1972). The adjacency principle and induced movement. Perception \& Psychophysics, 11, 309-314.

Gogel, W. C., \& MacCracken, P. J. (1979). Depth adjacency and induced motion. Perceptual \& Motor Skills, 48, 343-350.

Guerraz, M., Sakellari, V., Burchill, P., \& Bronstein, A. M. (2000). Influence of motion parallax in the control of spontaneous body sway. Experimental Brain Research, 131, 244-252.

HeYwood, S. (1973). Pursuing stationary dots: Smooth eye movements and apparent motion. Perception, 2, 181-195.

Howard, I. P., \& Rogers, B. J. (1995). Binocular vision and stereopsis. Oxford: Oxford University Press.

Isableu, B., Ohlmann, T., Crémieux, J., \& Amblard, B. (1998). Selection of spatial frame of reference and postural control variability. Experimental Brain Research, 114, 584-589.

JoNES, R. K., \& LEE, D. N. (1981). Why two eyes are better than one: The two views of binocular vision. Journal of Experimental Psychology: Human Perception \& Performance, 7, 30-40.

Lacour, M., Bathelemy, J., Borel, L., Magnan, J., Xerri, C., Chays, A., \& Ouaknine, M. (1997). Sensory strategies in human postural control before and after unilateral vestibular neurotomy. $E x$ perimental Brain Research, 115, 300-310.

LEE, D. N., \& Lishman, J. R. (1975). Visual proprioceptive control of stance. Journal of Human Movement Studies, 1, 87-95.

Lestienne, F., Schoechting, J. F., \& Berthoz, A. (1977). Postural readjustment induced by linear motion of visual scenes. Experimental Brain Research, 28, 363-384.

Masson, G., Mestre, D. R, \& Pailhous, J. (1995). Effects of the spatiotemporal structure of optical flow on postural readjustments in man. Experimental Brain Research, 103, 137-150.

Paulus, W. M., Straube, A., \& Brandt, T. (1984). Visual stabilization of posture: Physiological stimulus characteristics and clinical aspects. Brain, 107, 1143-1163.

Reinhardt-Rutland, A. H. (1988). Induced movement in the visual modality: An overview. Psychological Bulletin, 10, 57-71.

Riccio, G. E., \& Stoffregen, T. A. (1988). Affordances as constraints on the control of stance. Human Movement Science, 7, 265-300.

Stoffregen, T. A. (1985). Flow structure versus retinal location in the optical control of stance. Journal of Experimental Psychology: Human Perception \& Performance, 11, 554-565.

Stoffregen, T. A. (1986). The role of optical velocity in the control of stance. Perception \& Psychophysics, 39, 355-360.

Stoffregen, T. A, Smart, L. J., Bardy, B. G., \& Pagulayan, R. J. (1999). Postural stabilization of looking. Journal of Experimental Psychology: Human Perception \& Performance, 25, 1641-1658.

van Asten, W. N., Gielen, C. C., \& Diener van der Gon, J. J. (1988). Postural adjustments induced by simulated motion of differently structured environments. Experimental Brain Research, 73, 371-383.

WAESPE, W., \& SchWARz, U. (1987). Slow eye movements induced by apparent target motion in monkey. Experimental Brain Research, 67, 433-435.

Warren, W. H., Kay, B. A., \& Yilmaz, E. H. (1996). Visual control of posture during walking: Functional specificity. Journal of Experimental Psychology: Human Perception \& Performance, 22, 818-838.

YASui, S., \& Young, L. R. (1975). Perceived visual motion as effective stimulus to pursuit eye movement system. Science, 190, 906-908.

(Manuscript received April 27, 1999; revision accepted for publication May 10, 2000.) 